International Journal of Applied Mathematics

Volume 31 No. $3 \quad 2018,349-357$

ISSN: 1311-1728 (printed version); ISSN: 1314-8060 (on-line version)

doi: http://dx.doi.org/10.12732/ijam.v31i3.4

\title{
ON SATURATION ORDER OF FUNCTIONS OF SOME VARIABLES BY SINGULAR INTEGRALS
}

Ali M. Musayev

Azerbaijan State Oil and Industry University, Azadlig av. 20, AZ 1601, Baku, AZERBAIJAN

Abstract: In the paper we consider approximation of functions $f(x) \in$ $L^{p}\left(R_{n}\right)$, by $\alpha$-singular integrals, determine approximation order and saturation class.

AMS Subject Classification: 41A35, 42A38, 42B20

Key Words: singular integrals, one 0-dimensional kernels, Fourier transformation, Fejer operator

\section{Introduction}

Let $R_{n}$ be $n$ dimensional Euclidean space and $\lambda=\left(\lambda_{1}, \lambda_{2}, \ldots, \lambda_{n}\right)$, $t=\left(t_{1}, t_{2}, \ldots, t_{n}\right), K_{\lambda}(t)=\prod_{l=1}^{n} K_{l}, \lambda_{l}\left(t_{l}\right)$, where $K_{l, \lambda_{l}}\left(t_{l}\right)\left(t_{l} \in R_{1}, \lambda_{l}>0\right.$, $1 \leq l \leq n)$ are one-dimensional kernels satisfying the following conditions:

$$
\begin{gathered}
\frac{1}{\sqrt{2 \pi}} \int_{-\infty}^{+\infty} K_{l, \lambda_{l}}\left(t_{l}\right) d t_{l}=1, \quad\left\|K_{l, \lambda_{l}}\left(t_{l}\right)\right\|_{L\left(R_{1}\right)} \leq M_{l}<\infty, \\
\lim _{\lambda_{l} \rightarrow \infty} \int_{\left|t_{l}\right| \leq \delta}\left|K_{l, \lambda_{l}}\left(t_{l}\right)\right| d t_{l}=0
\end{gathered}
$$

for every $1 \leq l \leq n$. 
Consider for every $1 \leq l \leq n$, the $\alpha$-singular integral of the general form

$$
\begin{aligned}
& Q_{l, \lambda_{l}}^{(\alpha)}(f, x)=\frac{1}{(\sqrt{2 \pi})^{n}} \int_{R_{n}}\left\{\sum_{s_{1}, \ldots, s_{n}=1}^{\infty}\left[\prod_{l=1}^{n}(-1)^{s_{l}-1}\left(\begin{array}{c}
\alpha \\
s_{l}
\end{array}\right)\right]\right. \\
& \left.\times f\left(x_{1}-s_{1} t_{1}, \ldots, x_{n}-s_{n} t_{n}\right)\right\}\left[\prod_{l=1}^{n} K_{l, \lambda_{l}}\left(t_{l}\right)\right] d t_{1} \ldots d t_{n}
\end{aligned}
$$

where $\alpha>0$ is any real number, and one-dimensional kernels satisfy conditions (1).

Note that if $f(x) \in L^{p}\left(R_{n}\right) \quad(1 \leq p<\infty)$ and the kernel $K_{l, \lambda_{l}}\left(t_{l}\right)$ satisfies conditions (1), then singular integral (2) exists almost everywhere on $R_{n}$ and the following relations are valid:

a) $\left\|Q_{l, \lambda_{l}}^{(\alpha)}(f, x)\right\|_{L^{p}\left(R_{n}\right)} \leq C_{2}\|f(x)\|_{L^{p}\left(R_{n}\right)} \cdot \prod_{l=1}^{n}\left\|K_{l, \lambda_{l}}\left(t_{l}\right)\right\|_{L\left(R_{1}\right)} ;$

b) $\lim _{\lambda_{1} \rightarrow \infty}\left\|Q_{l, \lambda_{l}}^{(\alpha)}(f, x)-f(x)\right\|_{L^{p}\left(R_{n}\right)}=0$;

$$
\lambda_{n} \rightarrow \infty
$$

c) $\lim _{\lambda_{\mu} \rightarrow \infty}\left\|Q_{l, \lambda_{l}}^{(\alpha)}(f, x)-f(x)\right\|_{L^{p}\left(R_{n}\right)}=\left\|Q_{l, \lambda_{l}}^{(\alpha)}(f, x)-f(x)\right\|_{L^{p}\left(R_{n}\right)}$

for every $1 \leq \mu \leq n$ and $\lim _{\lambda_{\mu} \rightarrow \infty}$ means that $\lambda_{\mu} \rightarrow \infty$ for every $1 \leq \mu \leq n(\mu \neq l)$;

d)

$$
\left\|f(x)-Q_{l, \lambda_{l}}^{(\alpha)}(f, x)\right\|_{L^{p}\left(R_{n}\right)} \leq \sum_{\mu=1}^{n-1}\left\{\sum_{S_{1}, \ldots, S_{n}=1}^{\infty}\left[\prod_{l=\mu+1}^{n}\left(\begin{array}{l}
\alpha \\
s_{l}
\end{array}\right)\right]\right.
$$

$$
\left.\times\left[\prod_{l=\mu+1}^{n} K_{l, \lambda_{l}}\left(t_{l}\right)\right]_{L\left(R_{1}\right)}\right\}+\left\|f(x)-Q_{n, \lambda_{n}}^{(\alpha)}(f, x)\right\|_{L^{p}\left(R_{n}\right)} .
$$

In the sequel, we will assume

$$
G_{l, \lambda_{l}}^{(\alpha)}\left(u_{l}\right)=\sum_{S_{l}=1}^{\infty}(-1)^{s_{l}-1}\left(\begin{array}{c}
\alpha \\
s_{l}
\end{array}\right) K_{l, \lambda_{l}}^{\wedge}\left(u_{l} s_{l}\right)
$$

at $\lambda_{l}>0$ for every $1 \leq l \leq n$, where $K_{l, \lambda_{l}}^{\wedge}\left(u_{l}\right)$ is Fourier transformation of the functions $K_{l, \lambda_{l}}\left(t_{l}\right)$.

Denote by $F$ the set of all infinitely differentiable functions with a compact support. Introduce the class of functions

$$
M_{F}^{l}(\psi) \equiv\left\{\psi(x) \in F, \quad \eta_{l}\left(u_{l}\right) \psi^{\wedge}(u)\right\}=r_{\psi}^{\wedge}(u)
$$

for some $r_{\psi}(x) \in F, \quad \eta_{l}\left(u_{l}\right) \neq 0, \quad 1 \leq l \leq n$. 
Theorem 1. Let $f(x) \in L^{p}\left(R_{n}\right)(1 \leq p<\infty)$ and one-dimensional kernels $K_{l, \lambda_{l}}\left(t_{l}\right)\left(t_{l} \in R_{1}, \quad \lambda_{l}>0, \quad l=\overline{1, n}\right)$ of singular integrals (2) be such that the function

$$
\beta_{\lambda_{l}}^{(\alpha)}\left(u_{l}\right)=\frac{1-G_{l, \lambda_{l}}^{(\alpha)}\left(u_{l}\right)}{\tau_{l}\left(\lambda_{l}\right) \eta_{l}\left(u_{l}\right)} \quad\left(\tau_{l}\left(\lambda_{l}\right)>0, \quad \lim _{\lambda_{l} \rightarrow 0} \tau_{l}\left(\lambda_{l}\right)=0\right)
$$

be Fourier-Stieltjes transformation of some function

$$
\mu_{\lambda}^{(\alpha)}(t) \in N B V(-\infty ;+\infty)
$$

(i.e. $\frac{1}{\sqrt{2}} \int_{-\infty}^{\infty} d \mu_{\lambda_{l}}^{(\alpha)}(t)=1$ and $\int_{-\infty}^{\delta}+\int_{\delta}^{+\infty}\left|d \mu_{\lambda_{l}}^{(\alpha)}(t)\right| \rightarrow 0$ as $\left.\lambda_{l} \rightarrow \infty, \quad 1 \leq l \leq n\right)$.

Then:

I. if

$$
\left\|f(x)-Q_{l, \lambda_{l}}^{(\alpha)}(f, x)\right\|_{L^{p}\left(R_{n}\right)}=O \quad\left(\sum_{l=1}^{n} \tau_{l}\left(\lambda_{l}\right)\right)
$$

as $\lambda \rightarrow \infty$, then $f(x)=0$ almost everywhere on $R_{n}$;

II. The following relations are equivalent:

A)

$$
\left\|f(x)-Q_{l, \lambda_{l}}^{(\alpha)}(f, x)\right\|_{L^{p}\left(R_{n}\right)}=O \quad\left(\sum_{l=1}^{n} \tau_{l}\left(\lambda_{l}\right)\right)
$$

as $\lambda \rightarrow \infty$ (this means that $\lambda_{l} \rightarrow \infty$ for every $1 \leq l \leq n$ separately);

$B)$ there exist a bounded measure $v$ on $R_{n}$ and the function $l(x) \in L^{p}\left(R_{n}\right)$ such that for every $\psi(x) \in M_{F}^{l}(\psi)$ the following relation is valid

$$
\int_{R_{n}} r_{\psi}(x) f(x) d x= \begin{cases}\int_{R_{n}} \psi(x) d v(x) & \text { for } \quad p=1, \\ \int_{R_{n}} \psi(x) l(x) & \text { for } 1<p<\infty .\end{cases}
$$

Proof. Let us consider the case $1<p<\infty$.

I. According to c) we have

$$
\left\|f(x)-Q_{l, \lambda_{l}}^{(\alpha)}(f, x)\right\|_{L^{p}\left(R_{n}\right)}=O \quad\left(\tau_{l}\left(\lambda_{l}\right)\right) \quad(1 \leq l \leq n),
$$

as $\lambda_{l} \rightarrow \infty$. Then for any function $\psi(x) \in F$ we have

$$
\lim _{\lambda_{l} \rightarrow \infty} \int_{R_{n}} \frac{f(x)-Q_{l, \lambda_{l}}^{(\alpha)}(f, x)}{\tau_{l}\left(\lambda_{l}\right)} \psi(x) d x=O \quad(1 \leq l \leq n) .
$$


As the singular integral (2) is a convolution type integral, we find

$$
\int_{R_{n}} \frac{f(x)-Q_{l, \lambda_{l}}^{(\alpha)}(f, x)}{\tau_{l}\left(\lambda_{l}\right)} \psi(x) d x=\int_{R_{n}} \frac{\varphi(x)-Q_{l, \lambda_{l}}^{(\alpha)}(\psi, x)}{\tau_{l}\left(\lambda_{l}\right)} f(x) d x
$$

for every $\psi(x) \in F$.

Furthermore, from $\psi(x) \in M_{F}^{l}(\psi)$ and theorem on convolution of Fourier transformation, we have

$$
\begin{gathered}
{\left[\frac{f(x)-Q_{l, \lambda_{l}}^{(\alpha)}(f, x)}{\tau_{l}\left(\lambda_{l}\right)}\right]^{\wedge}(u)=\frac{1-Q_{l, \lambda_{l}}^{(\alpha)}\left(u_{l}\right)}{\tau_{l}\left(\lambda_{l}\right)} \psi^{\wedge}(u)} \\
=\left[\mu_{\lambda_{l}}^{(\alpha)}\left(t_{l}\right)\right]^{\vee}\left(u_{l}\right) r_{\psi}^{\wedge}(u)=\left[\frac{1}{\sqrt{2 \pi}} \int_{-\infty}^{\infty} r_{\psi}\left(x-t_{l}\right) d \mu_{\lambda_{l}}^{(\alpha)}\left(t_{l}\right)\right]^{\wedge}(u) .
\end{gathered}
$$

Hence by the uniqueness of the Fourier transformation, we find:

$$
\frac{\psi(x)-Q_{l, \lambda_{l}}^{(\alpha)}(f, x)}{\tau_{l}\left(\lambda_{l}\right)}=\frac{1}{\sqrt{2 \pi}} \int_{-\infty}^{\infty} r_{\psi}\left(x-t_{l}\right) d \mu_{\lambda_{l}}^{(\alpha)}\left(t_{l}\right) .
$$

From the last equality it follows that

$$
\begin{gathered}
\left\|\frac{\psi(x)-Q_{l, \lambda_{l}}^{(\alpha)}(f, x)}{\tau_{l}\left(\lambda_{l}\right)}-r_{\psi}(x)\right\|_{L^{p}\left(R_{n}\right)} \\
\leq \frac{1}{\sqrt{2 \pi}}\left\|r_{\psi}\left(x-t_{l}\right)-r_{\psi}(x)\right\|_{L^{p}\left(R_{n}\right)} d \mu_{\lambda_{l}}^{(\alpha)}\left(t_{l}\right) \rightarrow 0
\end{gathered}
$$

as $\lambda_{l} \rightarrow \infty \quad(1 \leq l \leq n)$.

Hence we have

$$
\lim _{\lambda_{l} \rightarrow \infty} \int_{R_{n}} \frac{\psi(x)-Q_{l, \lambda_{l}}^{(\alpha)}(\psi, x)}{\tau_{l}\left(\lambda_{l}\right)} f(x) d(x)=\int_{R_{n}} r_{\psi}(x) f(x) d x
$$

for $f(x) \in L^{p}\left(R_{n}\right)$.

Therefore by (7) and (8),

$$
\int_{R_{n}} r_{\psi}(x) f(x) d x=0
$$


for $r_{\psi}(x) \in F$. Hence we conclude, that $f(x)=0$ almost everywhere on $R_{n}$.

II. $\mathrm{A}) \Rightarrow \mathrm{B}$ ). Taking into account $\mathrm{c}$ ), from (4) we get

$$
\left\|f(x)-Q_{l, \lambda_{l}}^{(\alpha)}(f, x)\right\|_{L^{p}\left(R_{n}\right)}=O \quad\left(\tau_{l}\left(\lambda_{l}\right)\right) \quad\left(\lambda_{l} \rightarrow \infty\right) .
$$

Then by the theorem on weak compactness (see [4], p.16) there exist the function $l(x) \in L^{p}\left(R_{n}\right)$ and sequence of numbers $l_{i}$ $\left(\lim _{l_{i} \rightarrow \infty} \lambda_{l_{i}}=\infty\right)$ such that

$$
\lim _{l_{i} \rightarrow \infty} \int_{R_{n}} \frac{f(x)-Q_{l, \lambda_{l}}^{(\alpha)}(f, x)}{\tau_{l}\left(\lambda_{l}\right)} \psi(x) d(x)=\int_{R_{n}} \psi(x) l(x) d x
$$

for any function $\psi(x) \in F$.

As the singular integral (2) is a convolution type integral, then taking account (9), we find

$$
\begin{gathered}
\lim _{\lambda_{l} \rightarrow \infty} \int_{R_{n}} \frac{f(x)-Q_{l, \lambda_{l}}^{(\alpha)}(f, x)}{\tau_{l}\left(\lambda_{l}\right)} \psi(x) d(x) \\
=\lim _{\lambda_{l} \rightarrow \infty} \int_{R_{n}} \frac{\psi(x)-Q_{l, \lambda_{l}}^{(\alpha)}(\psi, x)}{\tau_{l}\left(\lambda_{l}\right)} f(x) d x=\int_{R_{n}} r_{\psi}(x) f(x) d x .
\end{gathered}
$$

Comparing (10) and (11), we have

$$
\int_{R_{n}} r_{\psi}(x) f(x) d x=\int_{R_{n}} \psi(x) l(x) d x
$$

i.e. B) is valid.

Now prove $B) \Rightarrow A$ ). As

$$
\begin{aligned}
Q_{l, \lambda_{l}}^{(\alpha)}(f)=\int_{R_{n}} \frac{\psi(x)-Q_{l, \lambda_{l}}^{(\alpha)}(\psi, x)}{\tau_{l}\left(\lambda_{l}\right)} f(x) d x \\
=\int_{R_{n}} \frac{f(x)-Q_{l, \lambda_{l}}^{(\alpha)}(f, x)}{\tau_{l}\left(\lambda_{l}\right)} \psi(x) d x=Q_{l, \lambda_{l}}^{(\alpha)}(\psi),
\end{aligned}
$$

then as in the proof of relation $b) \Rightarrow c$ ) of the theorem in [7], we have

$$
\frac{f(x)-Q_{l, \lambda_{l}}^{(\alpha)}(f, x)}{\tau_{l}\left(\lambda_{l}\right)}=\frac{1}{\sqrt{2 \pi}} \int_{-\infty}^{\infty} l\left(x+t_{l}\right) d \mu_{\lambda_{l}}^{(\alpha)}\left(t_{l}\right) \quad(1 \leq l \leq n),
$$


or

$$
\begin{gathered}
\left\|\frac{f(x)-Q_{l, \lambda_{l}}^{(\alpha)}(f, x)}{\tau_{l}\left(\lambda_{l}\right)}-r_{\psi}(x)\right\|_{L^{p}\left(R_{n}\right)} \\
\leq \frac{1}{\sqrt{2 \pi}}\|l(x)\|_{L^{p}\left(R_{n}\right)} \int_{-\infty}^{\infty}\left|d \mu_{\lambda_{l}}^{(\alpha)}\left(t_{l}\right)\right| \leq M .
\end{gathered}
$$

That is, independently of $\lambda_{l}(1 \leq l \leq n)$,

$$
\left\|f(x)-Q_{l, \lambda_{l}}^{(\alpha)}(f, x)\right\|_{L^{p}\left(R_{n}\right)}=O \quad\left(\tau_{l}\left(\lambda_{l}\right)\right) \quad\left(\lambda_{l} \rightarrow \infty\right) .
$$

Taking into account d) from the last equality we find

$$
\left\|f(x)-Q_{\lambda}^{(\alpha)}(f, x)\right\|_{L^{p}\left(R_{n}\right)}=O \quad\left(\sum_{l=1}^{n} \tau_{l}\left(\lambda_{l}\right)\right)
$$

as $\lambda \rightarrow \infty$, i.e. A) is valid.

The theorem has been proved for $1<p<\infty$. For $p=1$, it is proved in the same way.

Apply this theorem to Fejer's specific linear operator,

$$
\sigma_{\lambda}(f, x)=\frac{1}{\prod_{l=1}^{n}\left(2 \pi \lambda_{l}\right)} \int_{R_{n}} f(x-t) \prod_{l=1}^{n}\left(\frac{\sin \frac{1}{2} \lambda_{l} t_{l}}{\frac{1}{2} t_{l}}\right)^{2} d t
$$

in the case $1 \leq p \leq 2$. In this case $\alpha=1$ and

$$
K_{\lambda}=\frac{1}{\prod_{l=1}^{n}\left(2 \pi \lambda_{l}\right)} \prod_{l=1}^{n}\left(\frac{\sin \frac{1}{2} \lambda_{l} t_{l}}{\frac{1}{2} t_{l}}\right)^{2}=\prod_{l=1}^{n} K_{l, \lambda_{l}}\left(t_{l}\right) .
$$

Since

$$
\left[K_{l, \lambda_{l}}\left(t_{l}\right)\right]^{\wedge}\left(u_{l}\right)=\left\{\begin{array}{l}
\frac{1}{\sqrt{2 \pi}}\left(1-\frac{\left|u_{l}\right|}{\lambda_{l}}\right) \quad \text { for }\left|u_{l}\right|<\lambda_{l} \\
0 \quad \text { for }\left|u_{l}\right| \geq \lambda_{l}
\end{array}\right.
$$

then

$$
G_{l, \lambda_{l}}^{(\alpha)}\left(u_{l}\right)= \begin{cases}1-\frac{\left|u_{l}\right|}{\lambda_{l}} & \text { for }\left|u_{l}\right|<\lambda_{l} \\ 0 & \text { for }\left|u_{l}\right| \geq \lambda_{l} .\end{cases}
$$

Therefore, the functions $\tau_{l}\left(\lambda_{l}\right)=\frac{1}{\lambda_{l}}$ and $\eta_{l}\left(u_{l}\right)=\left|u_{l}\right|$ satisfy the relations 


$$
\frac{1-G_{l, \lambda_{l}}^{(\alpha)}\left(u_{l}\right)}{\tau_{l}\left(\lambda_{l}\right)\left|u_{l}\right|}=\left\{\begin{array}{l}
1 \\
\frac{\lambda_{l}}{u_{l}} \quad \text { for }\left|u_{l}\right|<\lambda_{l}
\end{array}\right.
$$

It is known [6] that

$$
\frac{1}{\sqrt{2 \pi}} \int_{-\infty}^{\infty} \sqrt{\frac{2}{\pi}}\left(\frac{\sin t_{l}}{t_{l}}-C_{i} t_{l}\right) e^{-t_{l} \frac{u_{l}}{\lambda_{l}}} d t_{l}=\left\{\begin{array}{lll}
1 & \text { for } & \left|u_{l}\right|<\lambda_{l} \\
\frac{\lambda_{l}}{u_{l}} & \text { for } & \left|u_{l}\right| \geq \lambda_{l}
\end{array}\right.
$$

where $C_{i} t_{l}=-\int_{t_{l}}^{\infty} \frac{\cos u}{u} d u$.

Introduce the function

$$
l_{\lambda_{l}}\left(t_{l}\right)=\lambda_{l} \sqrt{\frac{2}{\pi}}\left(\frac{\sin t_{l} \lambda_{l}}{t_{l}}-C_{i}\left(t_{l} \lambda_{l}\right)\right) .
$$

Since

$$
\begin{gathered}
\int_{-\infty}^{\infty}\left|l_{\lambda_{l}}\left(t_{l}\right)\right| d t_{l}=\lambda_{l} \sqrt{\frac{2}{\pi}} \int_{-\infty}^{\infty}\left|\frac{\sin t_{l} \lambda_{l}}{t_{l}}-C_{i}\left(t_{l} \lambda_{l}\right)\right| d t_{l} \\
=\sqrt{\frac{2}{\pi}} \int_{-\infty}^{\infty}\left|\frac{\sin t_{l}}{t_{l}}-C_{i} t_{l}\right| d t_{l} \leq M_{1}<\infty
\end{gathered}
$$

then $l_{\lambda_{l}}\left(t_{l}\right) \in L\left(R_{n}\right)$.

On the other hand, if by

$$
\mu_{\lambda_{l}}\left(t_{l}\right)=\int_{-\infty}^{t_{l}} l_{\lambda_{l}}\left(u_{l}\right) d u_{l} \quad(1 \leq l \leq u)
$$

we denote the uniformly bounded measure on $R_{1}$, then by

$$
\int_{-\infty}^{\infty} \frac{\sin t_{l}}{t_{l}} d t_{l}=\pi \text { and } \int_{-\infty}^{\infty} C_{i} t_{l} d t_{l}=0
$$

we get $\mu_{\lambda_{l}}(-\infty)=0, \quad \mu_{\lambda_{l}}(+\infty)=\sqrt{2 \pi}$ for all values of $\lambda_{l}$ and

$$
\left[\operatorname{Var} \mu_{\lambda_{l}}\left(t_{l}\right)\right]_{-\infty}^{\infty}=\int_{-\infty}^{\infty}\left|l_{\lambda_{l}}\left(u_{l}\right)\right| d u_{l} \leq M_{2}<\infty
$$

The comparison of (13) and (14) shows that the function 


$$
\frac{1-C_{l, \lambda_{l}}^{(\alpha)}\left(u_{l}\right)}{\tau_{l}\left(\lambda_{l}\right) \eta_{l}\left(u_{l}\right)}
$$

is Fourier-Stieltes transformation of normalized function with bounded variation.

Consequently, the conditions of the theorem are satisfied for Fejer's singular integral.

Therefore we have the following

Corollary 2. Let $f(x) \in L^{p}\left(R_{n}\right) \quad(1 \leq p \leq 2)$. Then for the relation

$$
\left\|\sigma_{\lambda}(f, x)-f(x)\right\|_{L^{p}}=O\left(\sum_{l=1}^{n} \frac{1}{\lambda_{l}}\right)
$$

to hold as $\lambda_{l} \rightarrow \infty$, it is necessary and sufficient that almost everywhere $f(x)=$ 0 on $R_{n}$.

Corollary 3. Let $f(x) \in L^{p}\left(R_{n}\right) \quad(1 \leq p \leq 2)$. For the relation

$$
\left\|\sigma_{\lambda}(f, x)-f(x)\right\|_{L^{p}\left(R_{n}\right)}=O\left(\sum_{l=1}^{n} \frac{1}{\lambda_{l}}\right)
$$

to hold as $\lambda_{l} \rightarrow \infty$, it is necessary and sufficient that $f(x) \in n_{p}(f)$, where

$$
n_{p}(f)=\left\{\begin{array}{l}
f(x) \in L\left(R_{n}\right) / f(x) \in B \bigcup\left(R_{n}\right), \quad \text { for } p=1, \\
f(x) \in L^{p}\left(R_{n}\right) / f(x) \in A C_{l o c}\left(R_{1}\right), \quad \text { with respect to } x_{l}, \\
u \frac{\partial f(x)}{\partial x_{l}} \in L^{p}\left(R_{n}\right), \quad(1 \leq p \leq 2, \quad 1 \leq l \leq n) .
\end{array}\right.
$$

\section{References}

[1] H. Berens and P. Butzer, On the best approximation for approximation for singular integrals by Laplase-transform methods, J. Approximation Theory, JSNMS, Birkhäuser (1964), 24-42.

[2] P. Butzer, R. Nessel, Fourier Analysis and Approximation, Vol. 1, New York and London, 1971, $553 \mathrm{p}$.

[3] R.G. Mamedov, Mellin Transformation and Approximation Theory, Baku, Elm, 1991, 272 p. 
[4] H. Berens and P. Butzer, Uber die Darstelling holomorpher Funktionendurch Laplace-und Laplace Stieltjes Integrale, Mat. Z., 81, 1963.

[5] Cynoymu (G. Sunouchi), Direct theorems in the theory of approximation, Acta Math., 20, No 3-4 (1969), 409-420.

[6] A.M. Musayev, To the question of approximation of functions by the Mellin type operators in the space $X_{\sigma_{1}, \sigma_{2}}\left(E^{+}\right)$. Proc. of IMM of NAS Azerbaijan, 28 (2008), 69-73.

[7] R.M. Rzaev, A.M. Musayev, On approximation of functions by Mellin singular integrals, Trans. of NAS of Azerbaycan, 32, No 1 (2012), 107-117.

[8] A.M. Musayev, Multiparameter approximation of function of general variables by singular integrals, Azerb. Techn. Univ. Baku, No 2 (2014), 212218. 
